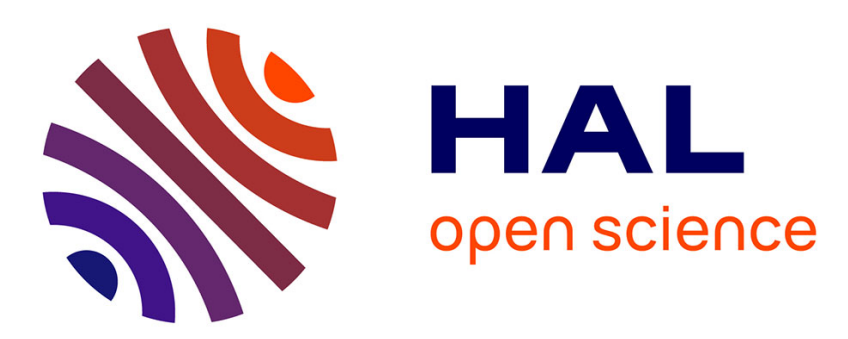

\title{
A Hybrid Distributed Strategy for Robust Global Phase Synchronization of Second-Order Kuramoto Oscillators
}

Alessandro Bosso, Ilario Antonio Azzollini, Simone Baldi, Luca Zaccarian

\section{To cite this version:}

Alessandro Bosso, Ilario Antonio Azzollini, Simone Baldi, Luca Zaccarian. A Hybrid Distributed Strategy for Robust Global Phase Synchronization of Second-Order Kuramoto Oscillators. CDC 2021 - 60th IEEE Conference on Decision and Control, Dec 2021, Austin, Texas, United States. 10.1109/CDC45484.2021.9682996 . hal-03370381

\section{HAL Id: hal-03370381 \\ https://hal.science/hal-03370381}

Submitted on 8 Oct 2021

HAL is a multi-disciplinary open access archive for the deposit and dissemination of scientific research documents, whether they are published or not. The documents may come from teaching and research institutions in France or abroad, or from public or private research centers.
L'archive ouverte pluridisciplinaire HAL, est destinée au dépôt et à la diffusion de documents scientifiques de niveau recherche, publiés ou non, émanant des établissements d'enseignement et de recherche français ou étrangers, des laboratoires publics ou privés. 


\title{
A Hybrid Distributed Strategy for Robust Global Phase Synchronization of Second-Order Kuramoto Oscillators
}

\author{
Alessandro Bosso ${ }^{1}$, Ilario A. Azzollini ${ }^{1}$, Simone Baldi $^{2}$, and Luca Zaccarian ${ }^{3}$
}

\begin{abstract}
This work proposes a distributed control strategy for the robust global leader-follower phase synchronization of Kuramoto oscillators with inertia. For a convenient design, the phase angles are represented as elements of the unit circle. In particular, we exploit a "half-angle" representation inspired by unit quaternions. The ensuing non-Euclidean state space poses some challenges for robust global stabilization, which can be conveniently overcome with dynamic hybrid feedback. For this reason, we propose a hybrid solution obtained by combining a distributed observer with local hysteresis-based tracking controllers. The overall closed-loop system is analyzed through reduction theorems and Lyapunov-based arguments.
\end{abstract}

\section{INTRODUCTION}

Numerous synchronization phenomena related to engineering, physics, and biology, can be naturally described through networks of coupled nonlinear oscillators. Among the mathematical models that capture synchronization phenomena, the Kuramoto model [1] is one of the most widespread. This model has received a renewed attention over the last years, mainly because of its applicability to neuronal synchronization [2] and synchronization in power networks [3], [4].

In networks of coupled oscillators, synchronization phenomena can either naturally arise, can be enforced by designing appropriate control laws, or can be promoted by a pacemaker in a leader-follower architecture [5]. In all these cases, several studies have been conducted on the various parameters that influence synchronization, such as the network's interaction topology, the diversity of the agents, and the coupling strengths [6], [7]. In particular, necessary [8] and sufficient [8], [9], [10] conditions for synchronization were found for Kuramoto networks.

This paper considers a second-order generalization of the classical first-order Kuramoto model, where each oscillator has its own inertia [4], [11]. Other generalizations have been recently proposed, such as the third-order model [12] and the Lohe model [13], which generalizes the Kuramoto model from the circle to the sphere.

\footnotetext{
${ }^{1}$ A. Bosso and I. A. Azzollini are with the Department of Electrical, Electronic, and Information Engineering (DEI), University of Bologna, Italy. Email: $\{a l e s s a n d r o . b o s s o, ~ i l a r i o . a z z o l l i n i\} @ u n i b o . i t$

${ }^{2} \mathrm{~S}$. Baldi is with the School of Mathematics, Southeast University, Nanjing, China, and the Delft Center for Systems and Control, Delft University of Technology, The Netherlands. Email: s.baldi@tudelft.nl

${ }^{3}$ L. Zaccarian is with LAAS-CNRS, Université de Toulouse, and the Department of Industrial Engineering, University of Trento, Italy. Email: zaccarian@laas.fr

S. Baldi was partly supported by the Double Innovation Plan Grant 4207012004 and the Special Funding for Overseas talents Grant 6207011901. L. Zaccarian was supported in part by ANR via Grant HANDY, number ANR-18-CE40-0010.
}

The synchronization of the Kuramoto model is an inherently nonlinear phenomenon. Since the oscillators' angular configurations are equivalent modulo $2 \pi$, their natural representation is given by elements of the unit circle. This leads to the $N$-torus as the state space of the network [14], [15]. Relevant challenges arise from the control viewpoint, mainly because the unit circle is not contractible, i.e., it is not diffeomorphic to any Euclidean space. This property is known to be a major obstacle to global stabilization. For instance, several works on rigid body control and estimation provide almost global results with continuous feedback [16], [17]. Global results can only be obtained via discontinuous feedback at the cost of sacrificing robustness. In this respect, hybrid systems theory has been proven successful for robust global stabilization on numerous non-contractible spaces, including unit quaternions [18] and the $N$-sphere [19].

Motivated by the above discussion, we present a hybrid distributed strategy for the robust global leader-follower phase synchronization of second-order Kuramoto oscillators. For simplicity, we assume that each node knows its local model parameters. To conveniently solve the problem, we introduce a new representation inspired by unit quaternions and based on "half-angle" expressions.

The proposed approach is composed of two main parts. First, the reference is estimated in each node of the network through a distributed observer, based on embedding the unit circle in $\mathbb{R}^{2}$. Then, employing the estimate thus obtained, we introduce a hysteresis-based hybrid controller inspired by [18] to guarantee global phase tracking for each oscillator. The resulting closed-loop dynamics can be described through a cascade of simple subsystems, each of them analyzed through Lyapunov theory. The overall stability analysis, performed through reduction theorems, not only shows globally robustly stable synchronization but also highlights that no time-scale separation and no tuning constraints are needed.

The paper is organized as follows. In Section II, we present the second-order Kuramoto model. Next, the control objectives are discussed in Section III, while in Sections IV and $\mathrm{V}$ we treat the distributed observer and the hysteresisbased controller. The interconnection of these components is then presented in Section VI. Finally, in Section VII, we validate the proposed strategy with a numerical example, while Section VIII provides some concluding remarks. Due to space constraints, some proofs are omitted.

Notation: We denote $\mathbb{R}_{\geq 0}:=[0, \infty)$, while $\mathbb{N}^{*}$ is the set of natural numbers zero excluded. Given column vectors $v$ and $w$, we often use $(v, w)$ to denote $\left[v^{\top} w^{\top}\right]^{\top}$. The notation $\otimes$ indicates the Kronecker product of matrices. For any $n \in \mathbb{N}^{*}$, 
$I_{n}$ is the identity matrix of dimension $n$ and $\mathbf{1}_{n} \in \mathbb{R}^{n}$ is the vector of all ones. Finally, we employ the hybrid systems formalism as in [20] and the graph theory formalism of [15].

\section{MODEL DESCRIPTION}

\section{A. Second-Order Kuramoto Network}

The second-order Kuramoto network is a system of $N$ nonlinear oscillators, coupled through an undirected and connected graph $\mathcal{G}:=\{\mathcal{V}, \mathcal{E}\}$, where for each node $i \in \mathcal{V}$, $\mathcal{N}_{i}$ is the set of its neighbors according to $\mathcal{E}$ :

$$
\begin{aligned}
\dot{\theta}_{i} & =\omega_{i}, \quad i \in \mathcal{V} \\
m_{i} \dot{\omega}_{i} & =-d_{i} \omega_{i}+\omega_{\mathrm{n} i}-\sum_{j \in \mathcal{N}_{i}} k_{i j} \sin \left(\theta_{i}-\theta_{j}-\varphi_{i j}\right)+u_{i} .
\end{aligned}
$$

In (1), for each $i \in \mathcal{V}, \theta_{i} \in \mathbb{R}$ and $\omega_{i} \in \mathbb{R}$ are the phase and the frequency, respectively, $u_{i}$ is the control input, $m_{i}>0$ is the oscillator's inertia, $d_{i}>0$ is a damping constant, whereas $\omega_{\mathrm{n} i}$ is the oscillator's natural frequency. Finally, $k_{i j}=k_{j i}>$ 0 and $\varphi_{i j}=\varphi_{j i} \in[0,2 \pi)$ are, respectively, the coupling weight and the phase shift between oscillators $i$ and $j$ (note that $\varphi_{i j}$ is often restricted to the set $[0, \pi / 2)$ ).

Define $\theta:=\left[\begin{array}{lll}\theta_{1} & \ldots & \theta_{N}\end{array}\right]^{\top}$ and $\omega:=\left[\begin{array}{lll}\omega_{1} & \ldots & \omega_{N}\end{array}\right]^{\top}$. With some abuse of notation, denote by $(\theta(\cdot), \omega(\cdot)): \mathbb{R}_{\geq 0} \rightarrow$ $\mathbb{R}^{2 N}$ a solution of system (1), for some input signal $u(\cdot)$ and with initial conditions $(\theta(0), \omega(0))$. We say that $(\theta(\cdot), \omega(\cdot))$ achieves phase synchronization if

$$
\lim _{t \rightarrow+\infty} \theta_{i}(t)-\theta_{j}(t) \in\{\tilde{\theta}: \tilde{\theta}=2 k \pi, k \in \mathbb{Z}\}, \quad \forall i, j \in \mathcal{V} .
$$

Similarly, the solution $(\theta(\cdot), \omega(\cdot))$ is said to achieve frequency synchronization if

$$
\lim _{t \rightarrow+\infty} \omega_{i}(t)-\omega_{j}(t)=0, \quad \forall i, j \in \mathcal{V}
$$

In this respect, the objective of this work consists in designing a distributed control strategy such that phase synchronization and convergence to a specified reference are reached globally, i.e., for any arbitrary initialization of the network (1). This goal is precisely defined in Section III.

\section{B. Quaternion-Inspired Representation}

In the following, we introduce a meaningful reformulation for system (1). Motivated by the equivalence modulo $2 \pi$ of angular values, our manipulations are based on the adoption of the unit circle $\mathbb{S}^{1}:=\left\{\left[\begin{array}{ll}\alpha & \beta\end{array}\right]^{\top} \in \mathbb{R}^{2}: \alpha^{2}+\beta^{2}=1\right\}$ as a more convenient representation of the phases $\theta_{i}$. Notably, $\mathbb{S}^{1}$ admits a Lie group structure, isomorphic to the group of planar rotations $\mathrm{SO}(2)$. In this respect, the function $\mathcal{R}$ : $\mathbb{S}^{1} \rightarrow \mathrm{SO}(2)$ maps any element $\left[\begin{array}{ll}\alpha & \beta\end{array}\right]^{\top} \in \mathbb{S}^{1}$ into the corresponding rotation matrix:

$$
\mathcal{R}\left(\left[\begin{array}{l}
\alpha \\
\beta
\end{array}\right]\right):=\left[\begin{array}{cc}
\alpha & -\beta \\
\beta & \alpha
\end{array}\right]
$$

which is used to compute the group multiplication as $\mathcal{R}(\gamma) \hat{\gamma}=\mathcal{R}(\hat{\gamma}) \gamma$, for any $\gamma, \hat{\gamma} \in \mathbb{S}^{1}$, with identity element

$$
e:=\left[\begin{array}{ll}
1 & 0
\end{array}\right]^{\top} \text {. }
$$

Employing the formalism just introduced, the representation adopted in [15] (also used in [21, Ex. 34]) is given by:

$$
\xi_{i}:=\left[\begin{array}{ll}
\cos \left(\theta_{i}\right) & \sin \left(\theta_{i}\right)
\end{array}\right]^{\top} \in \mathbb{S}^{1} .
$$

Through straightforward computations, with (6), the phase dynamics on both $\mathrm{SO}(2)$ and $\mathbb{S}^{1}$ is obtained as

$$
\frac{d}{d t} \mathcal{R}\left(\xi_{i}\right)=\omega_{i} J \mathcal{R}\left(\xi_{i}\right), \quad \dot{\xi}_{i}=\omega_{i} J \xi_{i}, \quad i \in \mathcal{V},
$$

where $J:=\left[\begin{array}{cc}0 & -1 \\ 1 & 0\end{array}\right] \in \mathrm{SO}(2)$.

Different from [15] and [21], we use here a second representation, which is more convenient for control design. Instead of (6), we represent $\theta_{i}$ through

$$
\zeta_{i}:=\left[\begin{array}{ll}
\eta_{i} & \epsilon_{i}
\end{array}\right]^{\top}:=\left[\begin{array}{ll}
\cos \left(\theta_{i} / 2\right) & \sin \left(\theta_{i} / 2\right)
\end{array}\right]^{\top} \in \mathbb{S}^{1} .
$$

More precisely, in place of $\xi_{i}$ in (6) we employ the element $\zeta_{i} \in \mathbb{S}^{1}$ such that $\xi_{i}=\phi\left(\zeta_{i}\right)$, where $\phi: \mathbb{S}^{1} \rightarrow \mathbb{S}^{1}$ is defined for any $\zeta_{i}=\left[\begin{array}{ll}\eta_{i} & \epsilon_{i}\end{array}\right]^{\top}$ as follows:

$$
\phi\left(\zeta_{i}\right):=\mathcal{R}\left(\zeta_{i}\right) \zeta_{i}=\left[\begin{array}{ll}
\eta_{i}^{2}-\epsilon_{i}^{2} & 2 \eta_{i} \epsilon_{i}
\end{array}\right]^{\top} .
$$

The map $\phi$ can be interpreted as the double-angle formula for elements on the unit circle. Note that $\phi$ is two-to-one, because $\phi\left(\zeta_{i}\right)=\phi\left(-\zeta_{i}\right)$. This property is analogous to the relation between rotation matrices in $\mathrm{SO}(3)$ and unit quaternions, whose domain, $\mathbb{S}^{3}$, is a double cover of $\operatorname{SO}(3)$. From $\xi_{i}=\phi\left(\zeta_{i}\right):=\mathcal{R}\left(\zeta_{i}\right) \zeta_{i}$, we obtain

$$
\dot{\xi}_{i}=\omega_{i} J \xi_{i}=\frac{\partial \phi}{\partial \zeta_{i}} \dot{\zeta}_{i}=2 \mathcal{R}\left(\zeta_{i}\right) \dot{\zeta}_{i},
$$

which leads to the phase dynamics in the new representation, presented both for $\mathrm{SO}(2)$ and $\mathbb{S}^{1}$ :

$$
\frac{d}{d t} \mathcal{R}\left(\zeta_{i}\right)=\frac{1}{2} \omega_{i} J \mathcal{R}\left(\zeta_{i}\right), \quad \dot{\zeta}_{i}=\frac{1}{2} \omega_{i} J \zeta_{i}, \quad i \in \mathcal{V} .
$$

Let $\mathbb{T}^{N}:=\prod_{i=1}^{N} \mathbb{S}^{1}$ denote the $N$-torus, then we can rewrite the network dynamics on $\mathbb{T}^{N} \times \mathbb{R}^{N}$ as follows:

$$
\begin{aligned}
\dot{\zeta}_{i}= & \frac{1}{2} \omega_{i} J \zeta_{i} \\
m_{i} \dot{\omega}_{i}= & -d_{i} \omega_{i}+\omega_{\mathrm{n} i}-\sum_{j \in \mathcal{N}_{i}} k_{i j} \phi\left(\zeta_{i}\right)^{\top} J \phi\left(\zeta_{j}\right) \cos \left(\varphi_{i j}\right) \\
& +\sum_{j \in \mathcal{N}_{i}} k_{i j} \phi\left(\zeta_{i}\right)^{\top} \phi\left(\zeta_{j}\right) \sin \left(\varphi_{i j}\right)+u_{i}, \quad i \in \mathcal{V} .
\end{aligned}
$$

In these new coordinates, we observe that condition (2) for phase synchronization coincides with

$$
\lim _{t \rightarrow+\infty} \mathcal{R}\left(\zeta_{i}(t)\right)^{\top} \zeta_{j}(t) \in\{-\boldsymbol{e}, \boldsymbol{e}\}, \quad \forall i, j \in \mathcal{V} .
$$

\section{Problem Statement}

\section{A. Leader Exosystem}

For simplicity, suppose that the graph $\mathcal{G}$, introduced for the physical couplings of network (12), also corresponds to the communication graph among the local controllers acting in each node. Then, we specify the tracking objectives through a leader system (sometimes indicated in the literature as pacemaker [5]) that delivers to the network some reference signals generated by the following autonomous exosystem:

$$
\left.\begin{array}{rl}
\dot{\zeta}^{\star} & =\frac{1}{2} c^{\top} w^{\star} J \zeta^{\star} \\
\dot{w}^{\star} & =S w^{\star}
\end{array}\right\} \quad\left(\zeta^{\star}, w^{\star}\right) \in \mathbb{S}^{1} \times \mathcal{W},
$$




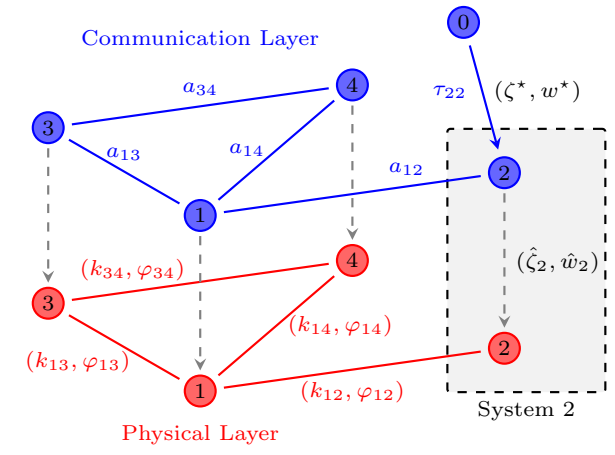

Fig. 1. Interaction and communication scheme.

where $\zeta^{\star}$ is the phase reference, $w^{\star} \in \mathbb{R}^{n}$ (with $n \in \mathbb{N}^{*}$ ) is used to generate the frequency reference $c^{\top} w^{\star}$, while $c \in \mathbb{R}^{n \times 1}$ and $S \in \mathbb{R}^{n \times n}$ are constant matrices. It is assumed that $S$ be Poisson stable (namely having only semisimple eigenvalues on the imaginary axis). Therefore, we can assume without loss of generality that the set $\mathcal{W} \subset$ $\mathbb{R}^{n}$ of admissible initial conditions $w^{\star}(0)$ is compact and forward invariant for $\dot{w}^{\star}=S w^{\star}$. Finally, we require the following connectivity property between the leader (14) and the network (12).

Assumption 1. The leader system (14) interacts, by communicating signal $\left(\zeta^{\star}, w^{\star}\right)$, with at least one node of $\mathcal{G}$.

Let $A=\left[a_{i j}\right] \in \mathbb{R}^{N \times N}$ and $L=\left[l_{i j}\right] \in \mathbb{R}^{N \times N}$ be the adjacency and Laplacian matrices of graph $\mathcal{G}$, and denote by $T=\left[\tau_{i j}\right] \in \mathbb{R}^{N \times N}$ the target matrix, a diagonal matrix such that $\tau_{i i}=1$ if (14) communicates with node $i \in \mathcal{V}$ and $\tau_{i i}=$ 0 otherwise. A scheme of the interaction and communication pattern is shown in Fig. 1.

The control problem of this work, stated below, corresponds to ensuring suitable stability and attractivity properties of the following synchronization set

$$
\begin{aligned}
& \mathcal{A}_{\mathrm{s}}:=\left\{\left(\zeta^{\star}, w^{\star}\right),\left(\zeta_{i}, \omega_{i}\right), i \in \mathcal{V}:\right. \\
&\left.\mathcal{R}\left(\zeta_{i}\right)^{\top} \zeta^{\star} \in\{-\boldsymbol{e}, \boldsymbol{e}\}, \omega_{i}=c^{\top} w^{\star}\right\},
\end{aligned}
$$

where $e$ is defined in (5). Note that $\mathcal{A}_{\mathrm{s}}$ is compact because (14) evolves in the compact set $\mathbb{S}^{1} \times \mathcal{W}$.

Problem 1. Consider the second-order Kuramoto network (12) and the exosystem (14). Let $m_{i}, d_{i}, \omega_{n i}, \varphi_{i j}, k_{i j}, c$, and $S$ be locally known at each node $i$. Design a distributed control strategy, based on the information exchange specified by $\mathcal{G}$ and Assumption 1, such that the network achieves global phase synchronization to the reference $\zeta^{\star}$. More specifically, design a distributed dynamic control strategy globally asymptotically stabilizing a compact set whose projection in the plant-exosystem direction coincides with the compact set $\mathcal{A}_{s}$ in (15).

The solution that we adopt for Problem 1 comprises hybrid dynamic laws whose data satisfy the so-called hybrid basic conditions of [20, Assumption 6.5]. As a consequence, our global asymptotic stability result is equivalent to the existence of a uniform class $\mathcal{K} \mathcal{L}$ bound (see [20, Thm. $7.12])$. Following the robustness results in $[20, \S 7.3]$, this also implies robust $\mathcal{K} \mathcal{L}$-asymptotic stability in the presence of fairly general perturbations of the dynamics. This property was not attained by using the discontinuous approach of [22].

\section{B. Control Architecture}

Through the parametrization (8), (9), (12), Problem 1 addresses the synchronization goal in (2), (3), in a convenient scenario wherein the set to be stabilized is compact. Topological limitations associated to the non-Euclidean nature of the phase dynamics make the solution to Problem 1 challenging. In fact, the $N$-torus is non-contractible, i.e., it is not diffeomorphic to any Euclidean space, and convergence to (15), as well as goal (2), require convergence to a disconnected set of points. Two main issues arise in this context. First of all, if the control laws $u_{i}$ are designed to stabilize only one of the given configurations, the trajectories in the coordinates $\xi_{i}$ (or $\theta_{i}$ ) will display the so-called unwinding phenomenon [18], causing unnecessary motion when the system is initialized close to synchronization. Additionally, if a static discontinuous feedback is adopted, the global attractivity property of the closed-loop system is not robust, e.g., to measurement noise. This fact, in practice, translates into chattering and high disturbance sensitivity [18].

Given these considerations, we employ the following hybrid dynamic strategy to robustly globally asymptotically stabilize a compact set containing $\mathcal{A}_{\mathrm{s}}$ in (15):

- first, we design a distributed observer for the exosystem (14), such that certain local estimates $\left(\hat{\zeta}_{i}, \hat{w}_{i}\right)$ of $\left(\zeta^{\star}, w^{\star}\right) \in \mathbb{S}^{1} \times \mathcal{W}$ are defined as elements of $\mathbb{R}^{2+n}$;

- for each agent $i$, we implement a hybrid mechanism for globally tracking $\left(\hat{\zeta}_{i}, \hat{w}_{i}\right)$. Exploiting the resulting cascade structure, the overall solution is analyzed via reduction theorems.

\section{Distributed ObSERVER Design}

We propose the following observer:

$$
\begin{aligned}
\dot{\hat{\zeta}}_{i} & =\frac{1}{2} c^{\top} \hat{w}_{i} J \hat{\zeta}_{i}-k_{\zeta} e_{\zeta_{i}} \quad i \in \mathcal{V}, \\
\dot{\hat{w}}_{i} & =S \hat{w}_{i}-k_{w} e_{w_{i}}
\end{aligned}
$$

where $\hat{\zeta}_{i} \in \mathbb{R}^{2}, \hat{w}_{i} \in \mathbb{R}^{n}$ are the local estimates of $\left(\zeta^{\star}, w^{\star}\right)$ at node $i, k_{\zeta}$ and $k_{w} \in \mathbb{R}$ are gains to be designed, while

$$
\begin{aligned}
& e_{\zeta_{i}}:=\sum_{j \in \mathcal{N}_{i}} a_{i j}\left(\hat{\zeta}_{i}-\hat{\zeta}_{j}\right)+\tau_{i i}\left(\hat{\zeta}_{i}-\zeta^{\star}\right) \\
& e_{w_{i}}:=\sum_{j \in \mathcal{N}_{i}} a_{i j}\left(\hat{w}_{i}-\hat{w}_{j}\right)+\tau_{i i}\left(\hat{w}_{i}-w^{\star}\right)
\end{aligned} \quad i \in \mathcal{V},
$$

are the local estimation errors, in which $a_{i j}$ and $\tau_{i i}$ are the entries of the adjacency matrix $A$ and the target matrix $T$.

To represent the overall network dynamics in a compact form, define the overall states $\hat{\zeta}:=\left[\hat{\zeta}_{1}^{\top} \ldots \hat{\zeta}_{N}^{\top}\right]^{\top} \in \mathbb{R}^{2 N}$ and $\hat{w}:=\left[\hat{w}_{1}^{\top} \ldots \hat{w}_{N}^{\top}\right]^{\top} \in \mathbb{R}^{N n}$ and the overall estimation errors $\tilde{\zeta}:=\hat{\zeta}-\mathbf{1}_{N} \otimes \zeta^{\star}$ and $\tilde{w}:=\hat{w}-\mathbf{1}_{N} \otimes w^{\star}$. Furthermore, define $e_{\zeta}:=\left[e_{\zeta_{1}}^{\top} \ldots e_{\zeta_{N}}^{\top}\right]^{\top} \in \mathbb{R}^{2 N}$ and $e_{w}:=\left[e_{w_{1}}^{\top} \ldots e_{w_{N}}^{\top}\right]^{\top} \in$ $\mathbb{R}^{N n}$, which from (17) can be written as [23]:

$$
e_{\zeta}=\left(B \otimes I_{2}\right) \tilde{\zeta}, \quad e_{w}=\left(B \otimes I_{n}\right) \tilde{w},
$$


where $B:=L+T$ is the leader-follower matrix, which is positive-definite since $T \neq 0$ from Assumption 1 (see [24]).

The dynamics of the frequency estimation error $\tilde{w}:=\hat{w}-$ $\mathbf{1}_{N} \otimes w^{\star}$ is obtained from (14), (16), (18) as

$$
\dot{\tilde{w}}=\left[\left(I_{N} \otimes S\right)-k_{w}\left(B \otimes I_{n}\right)\right] \tilde{w}
$$

leading to the following stability result.

Proposition 1. For any scalar gain $k_{w}>0$, the zeroequilibrium of system (19) is globally exponentially stable.

For notational convenience, define

$$
\tilde{\Omega}:=\operatorname{diag}\left(c^{\top} \tilde{w}_{1}, \ldots, c^{\top} \tilde{w}_{N}\right)=\operatorname{diag}\left(\left(I_{N} \otimes c^{\top}\right) \tilde{w}\right) .
$$

It follows that the dynamics of the phase estimation error $\tilde{\zeta}:=\hat{\zeta}-\mathbf{1}_{N} \otimes \zeta^{\star}$ is computed from (14), (16), (18) as:

$$
\begin{aligned}
\dot{\tilde{\zeta}}= & \left(\frac{1}{2} c^{\top} w^{\star}\left(I_{N} \otimes J\right)-k_{\zeta}\left(B \otimes I_{2}\right)+\frac{1}{2}(\tilde{\Omega} \otimes J)\right) \tilde{\zeta} \\
& +\frac{1}{2}(\tilde{\Omega} \otimes J)\left(\mathbf{1}_{N} \otimes \zeta^{\star}\right),
\end{aligned}
$$

with inputs $\zeta^{\star}, w^{\star}$, and $\tilde{w}$ (see (20)). The next proposition provides an input-to-state stability result for (21), used in Section VI to study the cascade between (19) and (21).

Proposition 2. For any scalar gain $k_{\zeta}>0$, system (21) is finite-gain exponentially input-to-state stable (ISS) with respect to the input $\tilde{w}$, uniformly in the inputs $\left(\zeta^{\star}, w^{\star}\right)$. Namely, there exist positive scalars $b, \lambda, \sigma$ such that, for any solution $\left(\zeta^{\star}(\cdot), w^{\star}(\cdot)\right)$ of the exosystem (14) and any $\tilde{w}(\cdot) \in \mathcal{L}_{\infty}$, the solutions of system (21) satisfy:

$$
|\tilde{\zeta}(t)| \leq b \exp (-\lambda t)|\tilde{\zeta}(0)|+\sigma\|\tilde{w}(\cdot)\|_{\infty}, \quad \forall t \in \mathbb{R}_{\geq 0} .
$$

\section{FULL-INFORMATION SYNCHRONIZATION}

In this section, we design a tracking controller in the simplified setup where the observer estimation errors are zero. Define the phase and frequency tracking errors

$$
\begin{aligned}
& \bar{\zeta}_{i}=\left[\begin{array}{ll}
\bar{\eta}_{i} & \bar{\epsilon}_{i}
\end{array}\right]^{\top}:=\mathcal{R}\left(\zeta_{i}\right)^{\top} \hat{\zeta}_{i} \in \mathbb{R}^{2} \quad i \in \mathcal{V} . \\
& \bar{\omega}_{i}:=c^{\top} \hat{w}_{i}-\omega_{i} \in \mathbb{R}
\end{aligned}
$$

In these coordinates, the control objective corresponds to imposing $\left(\bar{\epsilon}_{i}, \bar{\omega}_{i}\right) \rightarrow 0$, for all $i \in \mathcal{V}$. From (11), (12), (14), (16), we can compute the error dynamics as

$$
\begin{aligned}
\dot{\bar{\zeta}}_{i} & =\frac{1}{2} \bar{\omega}_{i} J \bar{\zeta}_{i}-k_{\zeta} \mathcal{R}\left(\zeta_{i}\right)^{\top} e_{\zeta_{i}} \quad i \in \mathcal{V}, \\
m_{i} \dot{\bar{\omega}}_{i} & =\psi_{i}-u_{i}-m_{i} k_{w} c^{\top} e_{w_{i}}
\end{aligned}
$$

where $\left(e_{\zeta_{i}}, e_{\omega_{i}}\right)$ are defined in (17) and:

$$
\begin{aligned}
\psi_{i}:= & d_{i} \omega_{i}-\omega_{\mathrm{n} i}+\sum_{j \in \mathcal{N}_{i}} k_{i j} \phi\left(\zeta_{i}\right)^{\top} J \phi\left(\zeta_{j}\right) \cos \left(\varphi_{i j}\right) \\
& -\sum_{j \in \mathcal{N}_{i}} k_{i j} \phi\left(\zeta_{i}\right)^{\top} \phi\left(\zeta_{j}\right) \sin \left(\varphi_{i j}\right)+m_{i} c^{\top} S \hat{w}_{i} .
\end{aligned}
$$

When $\hat{\zeta}_{i}=\zeta^{\star}$ and $\hat{w}_{i}=w^{\star}$ (i.e., $\left.(\tilde{\zeta}, \tilde{w})=0\right)$, the quantities in (23) become $\bar{\zeta}_{i}=\mathcal{R}\left(\zeta_{i}\right)^{\top} \zeta^{\star} \in \mathbb{S}^{1}$ and $\bar{\omega}_{i}=c^{\top} w^{\star}-\omega_{i} \in$ $\mathbb{R}$. In view of this reduction argument, we begin the design by assuming that the exosystem signals $\left(\zeta^{\star}, w^{\star}\right)$ are globally known for feedback. This full-information scenario corresponds to the requirement $T=I_{N}$, which will be removed in Section VI. Assuming that $\omega_{i}$ can be arbitrarily assigned by the controller, the phase dynamics in (24) reduces to

$$
\dot{\bar{\zeta}}_{i}=\frac{1}{2}\left(c^{\top} w^{\star}-\omega_{\mathrm{v} i}\right) J \bar{\zeta}_{i}, \quad i \in \mathcal{V}
$$

where $\omega_{\mathrm{v} i}$ is a virtual input. Define $\mathcal{Q}:=\{-1,1\}$ and choose any gain $k_{\mathrm{p}}>0$ and hysteresis margin $\delta \in(0,1)$. For each $i \in \mathcal{V}$, a hysteresis-based dynamic controller is given by

$$
\begin{aligned}
& \left\{\begin{array}{l}
\dot{q}_{i}=0, \quad\left(\bar{\zeta}_{i}, q_{i}\right) \in C_{\kappa} \\
q_{i}^{+}=-q_{i}, \quad\left(\bar{\zeta}_{i}, q_{i}\right) \in D_{\kappa}
\end{array} \quad i \in \mathcal{V}\right. \\
& \omega_{\mathrm{v} i}=c^{\top} w^{\star}+k_{\mathrm{p}} q_{i} \bar{\epsilon}_{i},
\end{aligned}
$$

where $q_{i} \in \mathcal{Q}$ is the controller state and

$$
\begin{aligned}
C_{\kappa} & :=\left\{\left(\bar{\zeta}_{i}, q_{i}\right) \in \mathbb{S}^{1} \times \mathcal{Q}: \bar{\eta}_{i} q_{i} \geq-\delta\right\} \\
D_{\kappa} & :=\left\{\left(\bar{\zeta}_{i}, q_{i}\right) \in \mathbb{S}^{1} \times \mathcal{Q}: \bar{\eta}_{i} q_{i} \leq-\delta\right\} .
\end{aligned}
$$

The closed-loop error dynamics then corresponds to

$$
\left\{\begin{array}{l}
\dot{\bar{\zeta}}_{i}=-\frac{1}{2} k_{\mathrm{p}} q_{i} \bar{\epsilon}_{i} J \bar{\zeta}_{i} \\
\dot{q}_{i}=0
\end{array} \quad\left[\begin{array}{l}
\bar{\zeta}_{i} \\
q_{i}
\end{array}\right] \in C_{\kappa},\left\{\begin{array}{l}
\bar{\zeta}_{i}^{+}=\bar{\zeta}_{i} \\
q_{i}^{+}=-q_{i}
\end{array}\left[\begin{array}{l}
\bar{\zeta}_{i} \\
q_{i}
\end{array}\right] \in D_{\kappa},\right.\right.
$$

which provides an autonomous hybrid dynamics having state $\left(\bar{\zeta}_{i}, q_{i}\right) \in \mathbb{S}^{1} \times \mathcal{Q}$ and such that the point $q_{i} \bar{\eta}_{i}=-1$ is not included in the flow set $C_{\kappa}$ (because $\delta<1$ ).

Lemma 1. The attractor $\mathcal{A}_{\kappa}:=\left\{(\zeta, q) \in \mathbb{S}^{1} \times \mathcal{Q}: \zeta=q \boldsymbol{e}\right\}$ is uniformly globally asymptotically stable (UGAS) for (29).

Controller (27), (28) can be employed in the design of the feedback laws $u_{i}$ for (24), following the energy-based approach proposed in [18]. Whenever $\hat{w}_{i}=w^{\star}$, due to our reduction argument, the dynamics of $\bar{\omega}_{i}$ in (24) reduces to

$$
m_{i} \dot{\bar{\omega}}_{i}=\psi_{i}-u_{i}, \quad i \in \mathcal{V},
$$

where $\psi_{i}$ in (25) is known to node $i$ (see Problem 1). The local controllers are given by

$$
\begin{aligned}
& \left\{\begin{array}{ll}
\dot{q}_{i}=0 & \left(\bar{\zeta}_{i}, q_{i}, \bar{\omega}_{i}\right) \in C:=C_{\kappa} \times \mathbb{R} \\
q_{i}^{+}=-q_{i} & \left(\bar{\zeta}_{i}, q_{i}, \bar{\omega}_{i}\right) \in D:=D_{\kappa} \times \mathbb{R}
\end{array} \quad i \in \mathcal{V}\right. \\
& u_{i}=\psi_{i}+k_{\mathrm{p}} q_{i} \bar{\epsilon}_{i}+k_{\mathrm{d}} \bar{\omega}_{i},
\end{aligned}
$$

with $C_{\kappa}$ and $D_{\kappa}$ as in (28) and where $k_{\mathrm{d}}$ is an arbitrary positive scalar. The closed-loop error dynamics for each node $i \in \mathcal{V}$ is then described by the following hybrid system

$$
\left\{\begin{array}{l}
\dot{\bar{\zeta}}_{i}=\frac{1}{2} \bar{\omega}_{i} J \bar{\zeta}_{i} \\
\dot{q}_{i}=0 \\
m_{i} \dot{\bar{\omega}}_{i}=-k_{\mathrm{p}} q_{i} \bar{\epsilon}_{i}-k_{\mathrm{d}} \bar{\omega}_{i}
\end{array} \quad\left[\begin{array}{c}
\bar{\zeta}_{i} \\
q_{i} \\
\bar{\omega}_{i}
\end{array}\right] \in C, \quad\left\{\begin{array}{l}
\bar{\zeta}_{i}^{+}=\bar{\zeta}_{i} \\
q_{i}^{+}=-q_{i} \\
\bar{\omega}_{i}^{+}=\bar{\omega}_{i}
\end{array} \quad\left[\begin{array}{c}
\bar{\zeta}_{i} \\
q_{i} \\
\bar{\omega}_{i}
\end{array}\right] \in D .\right.\right.
$$

The next result generalizes the argument of Lemma 1.

Proposition 3. For the full-information error dynamics (32), corresponding to (24), (31) with $(\tilde{\zeta}, \tilde{w})=0$, attractor $\mathcal{A}_{x}:=$ $\left\{(\zeta, q, \omega) \in \mathcal{S}^{1} \times \mathcal{Q} \times \mathbb{R}: \zeta=q \boldsymbol{e}, \omega=0\right\}$ is UGAS.

Proof: We sketch the proof inspired by [18]. Define $x_{i}:=\left(\bar{\zeta}_{i}, q_{i}, \bar{\omega}_{i}\right)$, then consider $V\left(x_{i}\right):=2 k_{\mathrm{p}}\left(1-q_{i} \bar{\eta}_{i}\right)+$ 


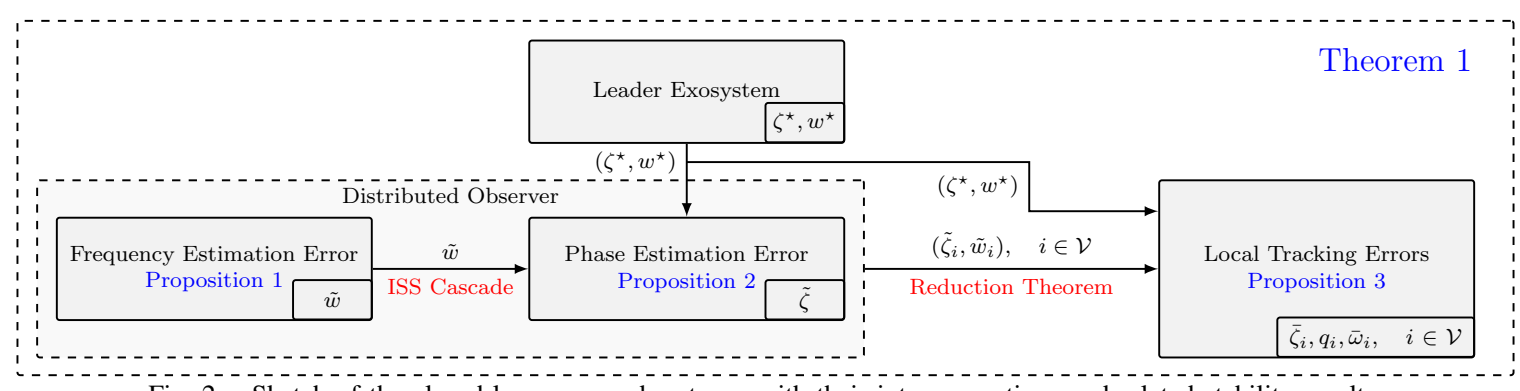

Fig. 2. Sketch of the closed-loop error subsystems, with their interconnections and related stability results.

$m_{i} \bar{\omega}_{i}^{2} / 2$, which is positive definite and radially unbounded w.r.t. $\mathcal{A}_{x}$. Then, straightforward computations show that

$$
\begin{array}{r}
\left\langle\nabla V\left(x_{i}\right), \dot{x}_{i}\right\rangle=-k_{\mathrm{d}} \bar{\omega}_{i}^{2} \leq 0, \quad \forall x_{i} \in C \backslash \mathcal{A}_{x} \\
V\left(x_{i}^{+}\right)-V\left(x_{i}\right)=4 k_{\mathrm{p}} q_{i} \bar{\eta}_{i} \leq-4 k_{\mathrm{p}} \delta<0, \quad \forall x_{i} \in D .
\end{array}
$$

It is then sufficient to notice that the largest invariant set contained in $\mathcal{S}=\left\{x_{i} \in C: \bar{\omega}_{i}=0\right\}$ satisfies $\bar{\epsilon}_{i}=0$ and $\bar{\eta}_{i} q_{i} \neq-1$, i.e., $V\left(x_{i}\right)=0$, and apply [20, Thm 8.8].

\section{Overall Control Scheme}

Finally, we present the complete hybrid observer-based controller for each node $i$, obtained by combining the distributed observer (16) and the local hysteresis-based controller (31). In this context, we no longer assume $(\tilde{\zeta}, \tilde{w})=0$, thus the dynamics of the tracking errors $\left(\bar{\zeta}_{i}, \bar{\omega}_{i}\right)$ in (24) is not simplified as in the full-information scenario.

Define the overall state at node $i$ as

$$
\chi_{i}:=\left(\hat{\zeta}_{i}, \hat{w}_{i}, \bar{\zeta}_{i}, q_{i}, \bar{\omega}_{i}\right) \in \mathbb{R}^{n+4} \times \mathcal{Q} \times \mathbb{R} .
$$

The controllers solving Problem 1 are given, for $i \in \mathcal{V}$, by

$$
\begin{aligned}
& \left\{\begin{array}{l}
\dot{\hat{\zeta}}_{i}=\frac{1}{2} c^{\top} \hat{w}_{i} J \hat{\zeta}_{i}-k_{\zeta} e_{\zeta_{i}} \\
\dot{\hat{w}}_{i}=S \hat{w}_{i}-k_{w} e_{w_{i}} \quad \chi_{i} \in C_{\chi},\left\{\begin{array}{l}
\hat{\zeta}_{i}^{+}=\hat{\zeta}_{i} \\
\hat{w}_{i}^{+}=\hat{w}_{i} \quad \chi_{i} \in D_{\chi}, \\
q_{i}^{+}=-q_{i}
\end{array}\right.
\end{array}\right. \\
& \text { with: } \quad C_{\chi}:=\left\{\chi_{i} \in \mathbb{R}^{n+4} \times \mathcal{Q} \times \mathbb{R}: \bar{\eta}_{i} q_{i} \geq-\delta\right\}, \\
& D_{\chi}:=\left\{\chi_{i} \in \mathbb{R}^{n+4} \times \mathcal{Q} \times \mathbb{R}: \bar{\eta}_{i} q_{i} \leq-\delta\right\},
\end{aligned}
$$

and: $u_{i}=\psi_{i}+k_{\mathrm{p}} q_{i} \bar{\epsilon}_{i}+k_{\mathrm{d}} \bar{\omega}_{i}$,

with $e_{\zeta_{i}}, e_{w_{i}}$ as in (17), $\bar{\eta}_{i}, \bar{\epsilon}_{i} \bar{\omega}_{i}$ defined in (23), and tuning parameters given by the stabilizer gains $k_{\mathrm{p}}>0, k_{\mathrm{d}}>0$, the observer gains $k_{\zeta}>0, k_{w}>0$, and the hysteresis margin $\delta \in(0,1)$. The next statement provides formal guarantees for the effectiveness of (35) in solving Problem 1.

Theorem 1. The attractor $\mathcal{A}$, corresponding to global phase synchronization to the reference $\zeta^{\star}$ and given by:

$$
\begin{gathered}
\mathcal{A}:=\left\{\left(\zeta^{\star}, w^{\star}, \chi_{1}, \ldots, \chi_{N}\right) \in \mathbb{S}^{1} \times \mathcal{W} \times\left(\mathbb{R}^{n+4} \times \mathcal{Q} \times \mathbb{R}\right)^{N}:\right. \\
\left.\hat{\zeta}_{i}=\zeta^{\star}, \hat{w}_{i}=w^{\star}, \bar{\zeta}_{i}=q_{i} e, \bar{\omega}_{i}=0, \forall i \in \mathcal{V}\right\},
\end{gathered}
$$

is globally robustly $\mathcal{K} \mathcal{L}$ asymptotically stable for the closedloop system, having state $\left(\zeta^{\star}, w^{\star}, \chi_{1}, \ldots, \chi_{N}\right)$ and obtained from the interconnection of the second-order Kuramoto network (12), the exosytem (14), and the local controllers (35).

Proof: The subsystems involved in the stability analysis are shown in Fig. 2, where we highlight the cascade structure of the closed-loop error dynamics.
Asymptotic stability of $\mathcal{A}$ is sketched through reduction theorems. By Propositions 1 and 2 and standard ISS results, the observer estimation error dynamics is UGAS. In other words, the closed (but not compact) attractor

$$
\begin{aligned}
\hat{\mathcal{A}}:=\left\{\left(\zeta^{\star}, w^{\star}, \chi_{1}, \ldots, \chi_{N}\right) \in \mathbb{S}^{1} \times \mathcal{W} \times\left(\mathbb{R}^{n+4} \times \mathcal{Q} \times \mathbb{R}\right)^{N}:\right. \\
\left.\hat{\zeta}_{i}=\zeta^{\star}, \hat{w}_{i}=w^{\star}, \forall i \in \mathcal{V}\right\},
\end{aligned}
$$

is UGAS. On the set $\hat{\mathcal{A}}$, we recover the full-information dynamics (32), thus, by Proposition $3, \mathcal{A}$ is UGAS relative to $\hat{\mathcal{A}}$. By [25, Cor 4.8$], \mathcal{A}$ is uniformly asymptotically stable for the overall closed-loop system, with basin of attraction given by all initial conditions generating bounded solutions.

Next, we show that all closed-loop solutions are bounded, which then implies UGAS. Note that the state $\left(\zeta^{\star}, w^{\star}\right)$ of (14) evolves in the bounded forward invariant set $\mathbb{S}^{1} \times \mathcal{W}$, thus it is bounded. Similarly, $q_{i}$ is bounded by construction. Due to Propositions 1 and $2,(\tilde{\zeta}, \tilde{w})$ converge to zero, therefore $\left(\hat{\zeta}_{i}, \hat{w}_{i}\right)$ are bounded for all $i \in \mathcal{V}$. We need to show that $\bar{\zeta}_{i}$ and $\bar{\omega}_{i}$ are bounded, for all $i \in \mathcal{V}$. Recall that $\bar{\zeta}_{i}:=\mathcal{R}\left(\zeta_{i}\right)^{\top} \hat{\zeta}_{i}$, thus $\bar{\zeta}_{i}$ is bounded as $\left|\bar{\zeta}_{i}\right| \leq\left|\hat{\zeta}_{i}\right|$. Regarding $\bar{\omega}_{i}$, let $V_{\omega}\left(\bar{\omega}_{i}\right):=m_{i} \bar{\omega}_{i}^{2} / 2$ and we have, using (24) and (35):

$$
\begin{aligned}
\dot{V}_{\omega} & =\bar{\omega}_{i}\left(-k_{\mathrm{d}} \bar{\omega}_{i}-k_{\mathrm{p}} q_{i} \bar{\epsilon}_{i}-m_{i} k_{w} c^{\top} e_{w_{i}}\right) \\
& \leq-\frac{k_{\mathrm{d}}}{2} \bar{\omega}_{i}^{2}+\frac{1}{2 k_{\mathrm{d}}}\left|k_{\mathrm{p}} q_{i} \bar{\epsilon}_{i}+m_{i} k_{w} c^{\top} e_{w_{i}}\right|^{2} \\
& \leq-\frac{k_{\mathrm{d}}}{m_{i}} V_{\omega}\left(\bar{\omega}_{i}\right)+\nu\left(\bar{\zeta}_{i}, \tilde{w}\right),
\end{aligned}
$$

where $\nu(\cdot)$ is bounded by a positive scalar $\bar{\nu}$ (depending on the initial conditions), because of the obtained bounds on $\bar{\zeta}_{i}$ and $\tilde{w}$. Since $\dot{V}_{\omega}<0$ for $V_{\omega}\left(\bar{\omega}_{i}\right)>m_{i} \bar{\nu} / k_{\mathrm{d}}$ and $V_{\omega}^{+}-V_{\omega}=0$, it follows that $\bar{\omega}_{i}$ is contained in a compact set, which proves global boundedness of solutions. The proof is completed by noticing that the closed-loop system satisfies the hybrid basic conditions, thus [20, Thm 7.21] ensures global robust $\mathcal{K} \mathcal{L}$ asymptotic stability.

\section{NumERICAL EXAMPLE}

We consider a second-order Kuramoto network with the communication and interaction structure as in Fig. 1, where $k_{12}=0.5, k_{13}=3, k_{14}=1, k_{34}=2, \varphi_{12}=\pi / 2$,

TABLE I

PARAMETERS AND INITIAL CONDITIONS OF THE OSCILLATORS

\begin{tabular}{c|c|c|c|c|c|} 
& $m_{i}$ & $d_{i}$ & $\omega_{\mathrm{n} i}$ & $\theta_{i}(0)$ & $\omega_{i}(0)$ \\
\hline \hline oscillator \#1 & 1.1 & 0.1 & 5 & $-\pi$ & 2 \\
oscillator \#2 & 1.3 & 0.15 & 10 & $\pi$ & 0.5 \\
oscillator \#3 & 1.2 & 0.2 & 15 & $\pi / 2$ & 1 \\
oscillator \#4 & 1.8 & 0.21 & 20 & $-\pi / 2$ & 0.3
\end{tabular}



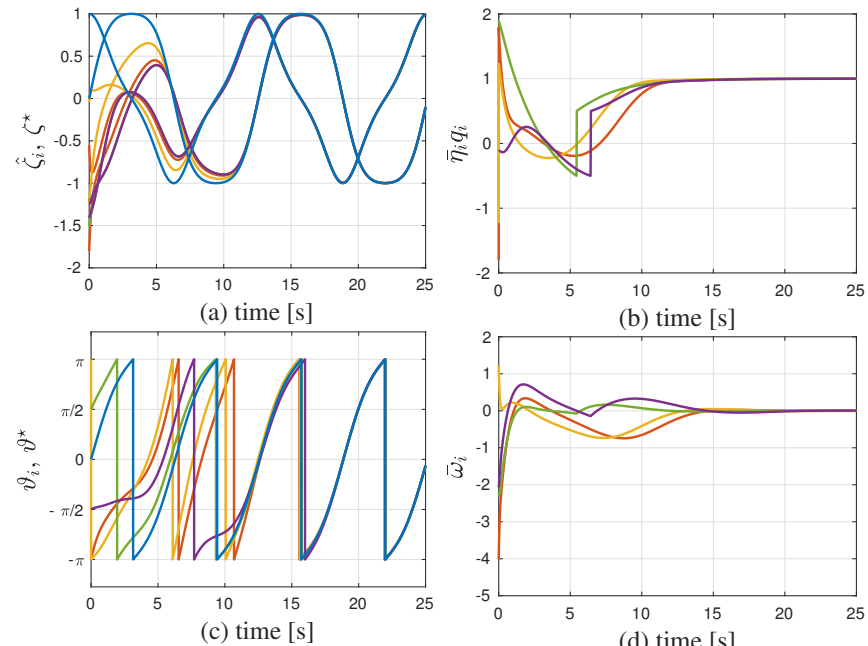

(b) time $[\mathrm{s}]$

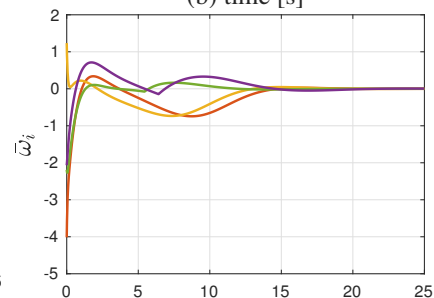

(d) time [s]

Fig. 3. Simulation results. (a): distributed observer phase estimation (reference in blue); (b): phase tracking errors; (c): phase angles represented in $[-\pi, \pi)$ (reference in blue); (d): frequency tracking errors.

$\varphi_{13}=\pi / 3, \varphi_{14}=\pi / 4, \varphi_{34}=\pi / 5$. We report in Tab. I the parameters and the initial conditions of each oscillator. The Kuramoto network has been implemented according to (1) and, in addition, each $\theta_{i}$ has been wrapped between $-2 \pi$ and $2 \pi$ to ensure boundedness of the simulation variables. For feedback computation, a continuous selection of $\zeta_{i}$ was obtained from (8). Exosystem (14) has been defined through:

$$
S=\left[\begin{array}{ccc}
0 & -1 & 0 \\
1 & 0 & 0 \\
0 & 0 & 0
\end{array}\right], \quad c^{\top}=\left[\begin{array}{lll}
0 & 1 & 1
\end{array}\right]
$$

with initial conditions $w^{\star}(0)=\left[\begin{array}{lll}0 & 0.5 & 1\end{array}\right]^{\top}$ and $\zeta^{\star}(0)=$ $\left[\begin{array}{ll}1 & 0\end{array}\right]^{\top}$. Instead, the controllers (35) have been initialized randomly, while the tuning parameters have been selected as $\delta=0.5, k_{\mathrm{p}}=k_{\mathrm{d}}=1, k_{\zeta}=2.5$, and $k_{w}=5$.

We provide in Fig. 3 the results of a simulation run, which confirms the effectiveness of the proposed solution. The quantities in subfigure (c) are computed as

$\vartheta^{\star}:=2 \operatorname{atan} 2\left(\zeta_{2}^{\star}, \zeta_{1}^{\star}\right), \quad \vartheta_{i}:=\bmod \left(\theta_{i}+\pi, 2 \pi\right)-\pi, i \in \mathcal{V}$,

where $\vartheta^{\star}$ is the angular reference corresponding to $\zeta^{\star}$, while $\vartheta_{i}$ represents $\theta_{i}$ in the interval $[-\pi, \pi)$.

\section{CONCLUSIONS}

We suggested a hybrid leader-follower phase synchronization control law for second-order Kuramoto oscillators. Under an undirected and connected graph topology, we provide an observer-stabilizer architecture, where the observer is distributed and the stabilizer exploits the local estimates of the reference motion, following a separation principle type of approach. Our main result, relying on hybrid reduction theorems, not only proves global convergence to the synchronization set but also its robust stability. Future work will provide an adaptive solution addressing unknown parameters in the dynamics of each oscillator.

\section{REFERENCES}

[1] Y. Kuramoto, Chemical Oscillations, Waves, and Turbulence. Springer, 1984.
[2] T. Menara, G. Baggio, D. S. Bassett, and F. Pasqualetti, "A framework to control functional connectivity in the human brain," in Proc. IEEE 58th Conf. Decis. Control, 2019, pp. 4697-4704.

[3] Y. Guo, D. Zhang, Z. Li, Q. Wang, and D. Yu, "Overviews on the applications of the Kuramoto model in modern power system analysis," Int. J. Electr. Power Energy Syst., vol. 129, p. 106804, 2021.

[4] F. Dörfler and F. Bullo, "Synchronization and transient stability in power networks and nonuniform Kuramoto oscillators," SIAM J. Control Optim., vol. 50, no. 3, pp. 1616-1642, 2012.

[5] Y. Wang and F. J. Doyle, "Exponential synchronization rate of Kuramoto oscillators in the presence of a pacemaker," IEEE Trans. Autom. Control, vol. 58, no. 4, pp. 989-994, April 2013.

[6] Y. Zhang and R. Xiao, "Synchronization of Kuramoto oscillators in small-world networks," Physica A: Stat. Mech. Appl., vol. 416, pp. 33 $-40,2014$.

[7] J. Zhang and J. Zhu, "Exponential synchronization of the highdimensional Kuramoto model with identical oscillators under digraphs," Automatica, vol. 102, pp. 122 - 128, 2019.

[8] N. Chopra and M. W. Spong, "On exponential synchronization of Kuramoto oscillators," IEEE Trans. Autom. Control, vol. 54, no. 2, pp. 353-357, Feb 2009.

[9] F. Dörfler and F. Bullo, "On the critical coupling for Kuramoto oscillators," SIAM J. Appl. Dynam. Syst., vol. 10, no. 3, pp. 10701099, 2011.

[10] L. Zhu and D. J. Hill, "Synchronization of Kuramoto oscillators: A regional stability framework," IEEE Trans. Autom. Control, vol. 65, no. 12, pp. 5070-5082, 2020.

[11] Y.-P. Choi, S.-Y. Ha, and S.-B. Yun, "Complete synchronization of Kuramoto oscillators with finite inertia," Physica D: Nonlinear Phenomena, vol. 240, no. 1, pp. $32-44,2011$.

[12] L. Wu and H. Chen, "Synchronization conditions for a third-order Kuramoto network," in Proc. 59th IEEE Conf. Decis. Control, 2020, pp. 5834-5839.

[13] J. Markdahl, D. Proverbio, and J. Goncalves, "Robust synchronization of heterogeneous robot swarms on the sphere," in Proc. 59th IEEE Conf. Decis. Control, 2020, pp. 5798-5803.

[14] L. Scardovi, A. Sarlette, and R. Sepulchre, "Synchronization and balancing on the N-torus," Syst. Contr. Lett., vol. 56, pp. 335-341, 2007.

[15] A. Bosso, I. A. Azzollini, and S. Baldi, "Global frequency synchronization over networks of uncertain second-order Kuramoto oscillators via distributed adaptive tracking," in Proc. IEEE 58th Conf. Decis. Control, 2019, pp. 1031-1036.

[16] R. Mahony, T. Hamel, and J.-M. Pflimlin, "Nonlinear complementary filters on the special orthogonal group," IEEE Trans. Autom. Control, vol. 53, no. 5, pp. 1203-1218, 2008.

[17] S. De Marco, L. Marconi, T. Hamel, and R. Mahony, "Output regulation on the special Euclidean group SE(3)," in Proc. IEEE 55th Conf. Decis. Control, 2016, pp. 4734-4739.

[18] C. G. Mayhew, R. G. Sanfelice, and A. R. Teel, "Quaternion-based hybrid control for robust global attitude tracking," IEEE Trans. Autom. Control, vol. 56, no. 11, pp. 2555-2566, 2011.

[19] P. Casau, C. G. Mayhew, R. G. Sanfelice, and C. Silvestre, "Robust global exponential stabilization on the n-dimensional sphere with applications to trajectory tracking for quadrotors," Automatica, vol. 110, p. 108534, 2019.

[20] R. Goebel, R. G. Sanfelice, and A. R. Teel, Hybrid Dynamical Systems: Modeling Stability, and Robustness. Princeton University Press, Princeton, NJ, 2012.

[21] _ - "Hybrid dynamical systems," IEEE Control Systems Magazine, vol. 29, no. 2, pp. 28-93, 2009.

[22] H. Gui and A. H. de Ruiter, "Global finite-time attitude consensus of leader-following spacecraft systems based on distributed observers," Automatica, vol. 91, pp. 225-232, 2018.

[23] I. A. Azzollini, W. Yu, S. Yuan, and S. Baldi, "Adaptive leader-follower synchronization over heterogeneous and uncertain networks of linear systems without distributed observer," IEEE Trans. Autom. Control, vol. 66, no. 4, pp. 1925-1931, 2020.

[24] H. Zhang and F. L. Lewis, "Adaptive cooperative tracking control of higher-order nonlinear systems with unknown dynamics," Automatica, vol. 48, no. 7, pp. 1432-1439, 2012.

[25] M. Maggiore, M. Sassano, and L. Zaccarian, "Reduction theorems for hybrid dynamical systems," IEEE Trans. Autom. Control, vol. 64, no. 6, pp. 2254-2265, 2018. 